\title{
Steady State Tokamak Equilibrium with Specified Magnetic Axis and Two Magnetic Null Points ${ }^{*}$
}

\author{
Osamu WATANABE \\ Advanced Numerical Analysis Center, Nagoya 468-0071, Japan
}

(Received 27 November 2012 / Accepted 4 March 2013)

\begin{abstract}
An analysis method of tokamak plasma equilibrium by a relaxation method with specified magnetic axis and null points (two magnetic separatrix points) is developed. The six degrees of freedom due to designated positions of the magnetic axis and null points is possible by using six poloidal field coil currents. Stable steady state tokamak plasma equilibria are calculated along with the MHD (magnetohydrodynamic) potential energy. Assuming an RF heating plasma, the plasma generates a plasma current which partially or fully cancels the magnetic field from the poloidal field coils. For low-temperature plasmas, the plasma current distribution is centrally peaked; for high-temperature plasmas, the plasma current has a hole. A centrally peaked current distribution in a low-temperature plasma is evolved into a current distribution with a hole by increasing the plasma pressure by heating. These calculations show that, under sufficient heating, the pressure driven current in tokamak plasmas form a current hole which minimizes the MHD potential energy.
\end{abstract}

(C) 2013 The Japan Society of Plasma Science and Nuclear Fusion Research

Keywords: tokamak plasma, equilibrium analysis, minimum MHD potential energy, pressure driven current, magnetic axis and magnetic null point specify

DOI: $10.1585 /$ pfr.8.2401026

\section{Introduction}

A current driven in a torus plasma is necessary for sustainment of a tokamak plasma to cancel charge separation. In spherical tokamaks, a tokamak plasma formation with a current drive can be achieved by RF heating alone [1-3]. Non-inductive tokamak plasma start-up and sustainment methods, including RF heating, reduce the role of central ohmic coils. The elimination of this central structure facilitates a fusion reactor design and provides possibilities of many plasma applications.

Judging from a tokamak plasma formation by RF heating alone, the tokamak formation is one of a transition to a minimum state of MHD potential energy. During start-up, a current drive mechanism that depends on a heating method might be the dominant factor determining the current distribution. After the start-up, it is presumed that a steady state tokamak has a current distribution which satisfies the condition of the minimum MHD potential energy state.

In addition, in order to sustain the steady state high performance tokamak plasma, the heat flux must be directed onto divertor tiles. As a result, a condition of the divertor field lines always restricts the equilibrium of the complete steady state high-temperature tokamak plasma. The shape and position of the divertor field line is closely related to the position of two null points (two magnetic separatrix points). For this reason, in this paper, we take

author'se-mail: rwata@cocoa.ocn.ne.jp

*) This article is based on the presentation at the 22nd International Toki Conference (ITC22). the positions of the null points to be specified. The shape of magnetic surfaces of the equilibrium tokamak are controllable by poloidal field coil currents, and we also take the position of the magnetic axis to be specified.

The functional form of the plasma pressure is assumed to be the sum of arbitrary functions of a magnetic surface function $\Psi$. The steady state coefficients for the linear combination are selected, after repeated equilibrium calculations, by the condition that they minimize the MHD potential energy $W_{\mathrm{M}}$;

$$
W_{\mathrm{M}}=\int \mathrm{d} V\left[\frac{P}{\gamma-1}+\frac{1}{2 \mu_{0}} \boldsymbol{B}^{2}\right] \equiv W_{\mathrm{P}}+W_{\mathrm{B}},
$$

where, $\gamma=5 / 3$ is the ratio of specific heats, $P$ is the plasma pressure, $\boldsymbol{B}$ is the magnetic field, $W_{\mathrm{P}}$ is the plasma stored energy, $W_{\mathrm{B}}$ is the magnetic energy, and $\mu_{0}$ is the vacuum permeability.

The equilibrium calculation for establishment of a new analytical method is performed with the parameters of JT-60SA [4]. In this method, the magnetic axis and two null points of the tokamak plasma are specified for the computation. The six poloidal field coil currents (EF coil currents of JT-60SA) can be determined uniquely by the pressure profile $P$. The center solenoid coil currents are useful for stabilization of the plasma. This can be expressed using the $\delta W_{\mathrm{M}}<0$ equilibrium formation;

$$
\delta W_{\mathrm{M}} \equiv \frac{W_{\mathrm{M}}-W_{\mathrm{M} 0}}{W_{\mathrm{M} 0}}, \quad W_{\mathrm{M} 0}=\int \mathrm{d} V \frac{\boldsymbol{B}_{0}^{2}}{2 \mu_{0}},
$$

where $\boldsymbol{B}_{0}$ is magnetic field without the plasma current. Plasma disruption can be avoided by heating, when $\delta W_{\mathrm{M}}<$ 
0 . The role of the center solenoid coils for the plasma stability is discussed in Sec. 3. Equilibrium is calculated for specified magnetic axis and null point positions using the Biot-Savart law to determine the magnetic field generated by the plasma current. The plasma current in the equilibrium is calculated using the ideal MHD equations. Any current drive mechanism, such as one originated from a special structure of RF antennas is not included in the calculation. The calculation method is presented in Sec. 2.

Several calculations are presented in Sec. 3. In these calculations, the coil currents are calculated as the plasma temperature is raised from $5 \mathrm{eV}$ to $20 \mathrm{keV}$, with the positions of the magnetic axis and null points assumed to be fixed. This calculation is useful for determination of a start-up scenario. This method can also be used in design of new experimental devices.

\section{Calculation Method}

The calculation method assumes equilibrium in a steady state tokamak with axisymmetric MHD equations in cylindrical coordinates $(r, \phi, z)$. The force balance in the direction of the magnetic field is derived from the MHD equations and depends on the pressure $P$, which is a function of a magnetic surface function $\Psi$, as $P=P(\Psi)$. The force balance in the direction of the current is represented using the toroidal magnetic field $B_{\phi}$, which depends on an arbitrary current function $I(\Psi)$, as $2 \pi r B_{\phi}=\mu_{0} I(\Psi)$. From a force balance perpendicular to the magnetic surface, the toroidal current is expressed by gradient terms of the pressure and the current function as follows.

$$
\begin{aligned}
0 & =\nabla \Psi \cdot(-\nabla P+\boldsymbol{J} \times \boldsymbol{B}) \\
& \Rightarrow J_{\phi}=r \frac{\mathrm{d} P(\Psi)}{\mathrm{d} \Psi}+\frac{\mu_{0} I(\Psi)}{4 \pi^{2} r} \frac{\mathrm{d} I(\Psi)}{\mathrm{d} \Psi} .
\end{aligned}
$$

Thus, the plasma current $\boldsymbol{J}$ is expressed as follows.

$$
\boldsymbol{J}=r \frac{\mathrm{d} P(\Psi)}{\mathrm{d} \Psi} \boldsymbol{e}_{\phi}+\frac{1}{2 \pi} \frac{\mathrm{d} I(\Psi)}{\mathrm{d} \Psi} \boldsymbol{B} .
$$

Here, $\boldsymbol{e}_{\phi}$ is unit vector in the toroidal direction. Equation (4) indicates that the gradient of the current function $I(\Psi)$ drives the plasma current along the magnetic field as indicated by the second term on the right-hand side (RHS). In this paper, we analyze the case of the current function being constant: $I(\Psi)=I_{0}$.

The magnetic surface function $\Psi$ is calculated from the Biot-Savart law. From the vector potential $\boldsymbol{A}(r, z)=$ $\mu_{0} / 4 \pi \int \mathrm{d}^{3} r^{\prime} \boldsymbol{J}\left(r^{\prime}\right) /\left|r^{\prime}-r\right|$, the magnetic surface function $\Psi$ of the equilibrium is determined by the following integral equation;

$$
\begin{aligned}
& \Psi(r, z)=\frac{r \mu_{0}}{4 \pi} \int r^{\prime} \mathrm{d} r^{\prime} \mathrm{d} \phi^{\prime} \mathrm{d} z^{\prime} \\
& \times\left[\frac{\left.\cos \phi^{\prime}\left\{r^{\prime} \frac{\mathrm{d} P(\Psi)}{\mathrm{d} \Psi}+\frac{\mu_{0} I(\Psi)}{4 \pi^{2} r^{\prime}} \frac{\mathrm{d} I(\Psi)}{\mathrm{d} \Psi}\right\}\right|_{\Psi=\Psi\left(r^{\prime}, z^{\prime}\right)}}{\sqrt{r^{\prime 2}-2 r r^{\prime} \cos \phi^{\prime}+r^{2}+\left(z-z^{\prime}\right)^{2}}}\right] \\
& +\Psi^{\mathrm{ext}}(r, z),
\end{aligned}
$$

where, $\Psi^{\text {ext }}(r, z)$ is a contribution from the external poloidal field coil currents.

We introduce a grid structure $(i, j)$ in the $r z$-plane of Eq. (5) and use a body current coil model for the plasma current in each unit grid cell. As the result, Eq. (5) can be integrated with $\left(\mathrm{d} r^{\prime}, \mathrm{d} z^{\prime}\right)$ analytically, and is reduced to an integral equation without a numerical divergence, as follows.

$$
\begin{gathered}
\begin{array}{c}
\Psi(r, z)=\frac{r \mu_{0}}{4 \pi} \sum_{i, j} J_{\phi_{i, j}} \int_{0}^{2 \pi} \mathrm{d} \phi^{\prime} \cos \phi^{\prime} \\
\times\left\{\left(z_{i, j}-z\right)\left(\frac{R}{2}+r \cos \phi^{\prime} F_{1}\right)\right. \\
\left.-\frac{r^{2} \sin 2 \phi^{\prime}}{2} F_{0}+\frac{r_{i, j}^{2}-r^{2} \cos 2 \phi^{\prime}}{2} F_{2}\right\}
\end{array} \\
R=\sqrt{r_{i, j}^{2}-2 r r_{i, j} \cos \phi^{\prime}+r^{2}+\left(z-z_{i, j}\right)^{2}} \\
F_{0}=\tan ^{-1} \frac{\left(r_{i, j}-r \cos \phi^{\prime}\right)\left(z_{i, j}-z\right)}{r \sin \phi^{\prime} R}, \\
F_{1}=\sinh ^{-1} \frac{r_{i, j}-r \cos \phi^{\prime}}{\sqrt{r^{2} \sin \phi^{\prime 2}+\left(z-z_{i, j}\right)^{2}}} \\
F_{2}=\sinh ^{-1} \frac{z_{i, j}-z}{\sqrt{r_{i, j}^{2}-2 r r_{i, j} \cos \phi^{\prime}+r^{2}}}
\end{gathered}
$$

Equation (6) can be solved by a relaxation method.

For the calculation in this paper, the positions of the magnetic axis $\left(r_{\mathrm{ax}}, z_{\mathrm{ax}}\right)$ and null points $\left(r_{\mathrm{sep}}^{ \pm}, z_{\mathrm{sep}}^{ \pm}\right)$are specified when solving for the tokamak equilibrium.

$$
B_{r}(r, z)=B_{z}(r, z)=0, \quad\left\{\begin{array}{cl}
r=r_{\mathrm{ax}}, & z=z_{\mathrm{ax}} \\
r=r_{\mathrm{sep}}^{ \pm}, & z=z_{\mathrm{sep}}^{ \pm}
\end{array}\right.
$$

where the superscripts \pm represent the upper and lower null points.

A pressure profile is assumed as follows.

$$
\begin{aligned}
P(\Psi)=2 T_{0} & {\left[n_{1} \exp \left\{\frac{1}{\sigma_{1}}\left(\frac{\Psi-\Psi_{\mathrm{ax}}}{\Psi_{\mathrm{ax}}-\Psi_{\mathrm{sep}}}\right)\right\}\right.} \\
& +n_{2} \exp \left\{-\frac{1}{\sigma_{2}}\left(\frac{\Psi-\Psi_{\mathrm{ax}}}{\Psi_{\mathrm{ax}}-\Psi_{\mathrm{sep}}}\right)^{2}\right\} \\
& +n_{3} \exp \left\{\frac{1}{\sigma_{3}}\left(\frac{\Psi-\Psi_{\mathrm{ax}}}{\Psi_{\mathrm{ax}}-\Psi_{\mathrm{sep}}}\right)^{3}\right\} .
\end{aligned}
$$

Where, $\Psi_{\text {ax }}$ and $\Psi_{\text {sep }}$ are the flux function $\Psi$ at magnetic axis and separatrix ( $\Psi_{\text {sep }} \leq \Psi \leq \Psi_{\text {ax }}$ : inside the plasma). $T_{0}$ is a plasma temperature. $\sigma_{1}, \sigma_{2}$ and $\sigma_{3}$ are set to 0.28 , $(0.48)^{2}$ and $(0.619)^{3}$, respectively. As the result, it is ensured that the plasma pressure on the separatrix $\left(\Psi=\Psi_{\text {sep }}\right)$ is less than 3 percent of the center one. The total plasma density on the magnet axis $n_{1}+n_{2}+n_{3}$ is assumed to be $10^{20} \mathrm{~m}^{-3}$, in this paper. In the RHS of Eq. (12), the first term gives a centrally peaked current profile, and the second and third terms give a current profile with a current hole. After the equilibrium calculation is converged, the 


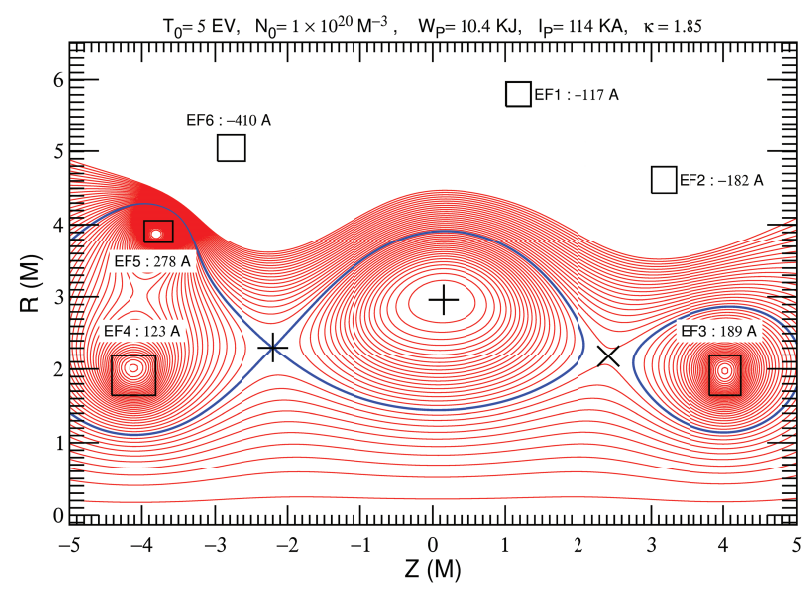

Fig. 1 Magnetic flux surface with $T_{0}=5 \mathrm{eV}$. The specified magnetic axis and null points are marked by crosses. The center solenoid coils CS have no current.

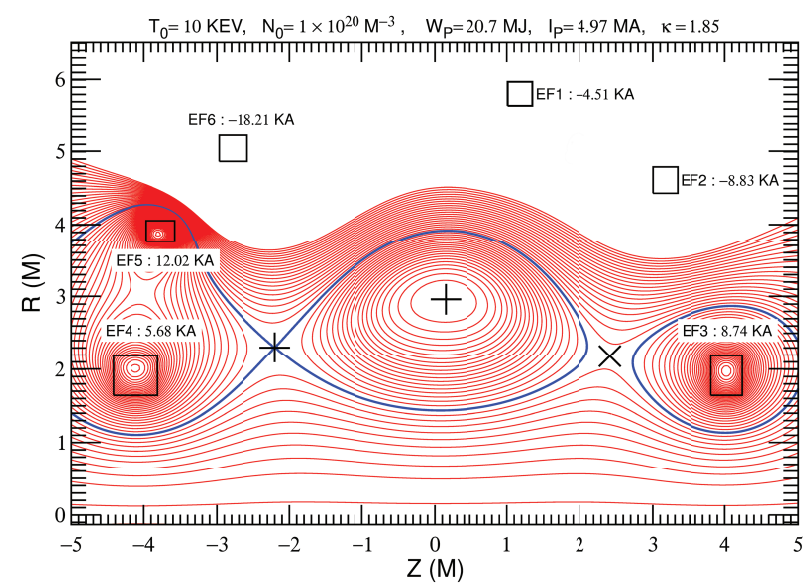

Fig. 2 Magnetic flux surface with $T_{0}=10 \mathrm{keV}$. Compared to Fig. 1, plasma current $I_{\mathrm{p}}$, coil currents and stored energy $\mathrm{W}_{\mathrm{p}}$ are increased significantly. But shapes of the magnetic flux surface with the elongation $\kappa$ look nearly identical. The center solenoid coils have no current.

MHD potential energy Eq. (1) is calculated and examined for plasma stability.

\section{Calculation Results}

Tokamak equilibria has been calculated based on the parameters of JT-60SA [4]. The plasma current outside the last closed flux surface is taken to be zero in the calculation. The device has six poloidal field coils from EF1 to EF6 and four center solenoid coils CS. Constant currents are driven in the center solenoid coils, but they are not used for the inductive current drive.

First, we consider equilibria without center solenoid coils. Figures 1 and 2 show the magnetic flux surfaces for the case of $T_{0}=5 \mathrm{eV}$ and $10 \mathrm{keV}$ respectively, with a plasma density on the magnetic axis $n_{1}=1 \times 10^{20} \mathrm{~m}^{-3}$, with $n_{2}=n_{3}=0$. Observe that, even though the pressures are very different, nearly identical magnetic flux surfaces

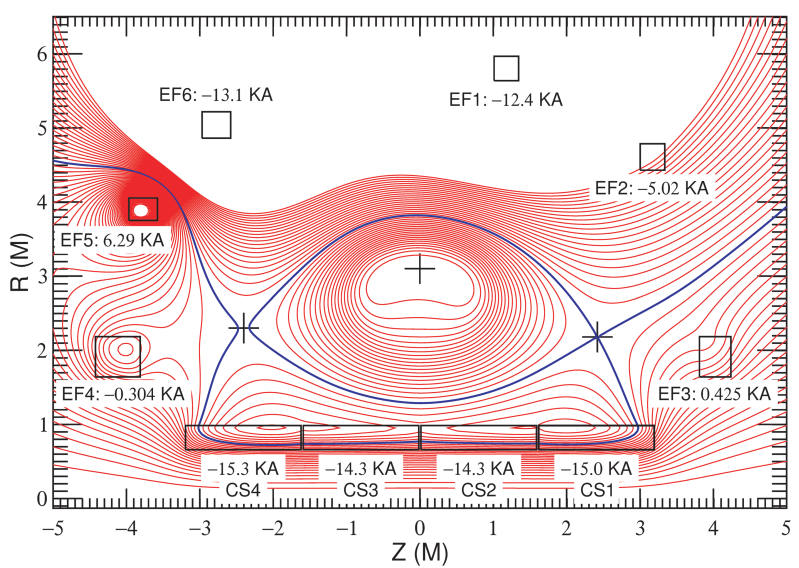

Fig. 3 Magnetic flux surface at $T_{0}=20 \mathrm{keV}$ with finite currents of four center solenoid coils. The plasma density is $n_{3}=$ $1 \times 10^{20} \mathrm{~m}^{-3}$ and $n_{1}=n_{2}=0$. A current hole is formed as shown in Fig. 4. A constant current is driven in the four center solenoid coils.

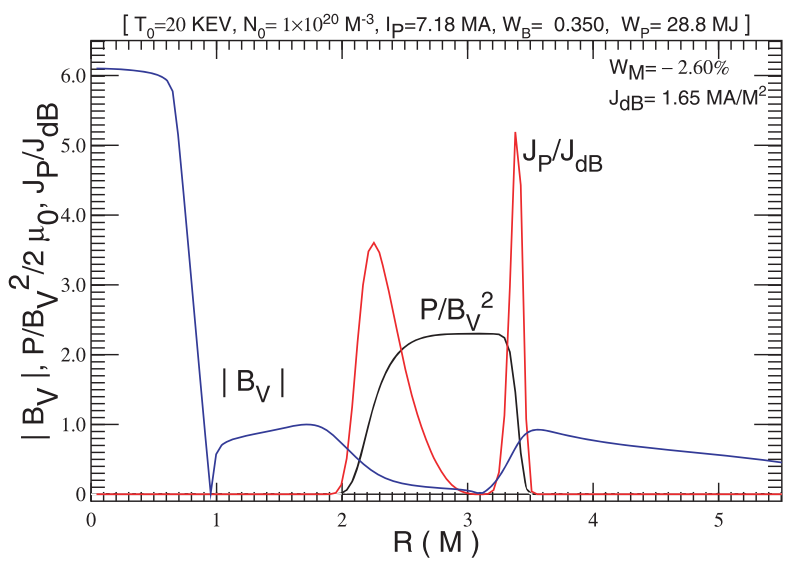

Fig. 4 Radial profiles of the plasma pressure $P$ and toroidal current density $J_{\mathrm{p}}$ at the mid-plane. $J_{\mathrm{dB}}$ is the poloidal diamagnetic current. $B_{\mathrm{v}}$ is a vertical magnetic field. The profile of the magnetic flux surface is shown in Fig. 3.

are obtained by adjusting the current in the poloidal field coils. Though the stored energy is increased, due to fixing the magnetic axis and null point positions, the tokamak plasma can keep its shape. In this case, the current direction in the EF3-5 coils are co-directional with the plasma current.

Next, we consider an equilibrium with the center solenoid coils. Figure 3 shows the magnetic flux surface of a high energy tokamak plasma $\left(T_{0}=20 \mathrm{keV}\right)$ in which a current hole is formed. Radial profiles are shown in Fig. 4. Because the magnetic field generated by the center solenoid coils is partially canceled by the magnetic field due to the plasma current, the plasma is stabilized and the MHD potential energy is reduced;

$$
\delta W_{\mathrm{M}}=-2.6015 \times 10^{-2} .
$$

Steady state current and pressure profiles in a $10 \mathrm{eV}$ 


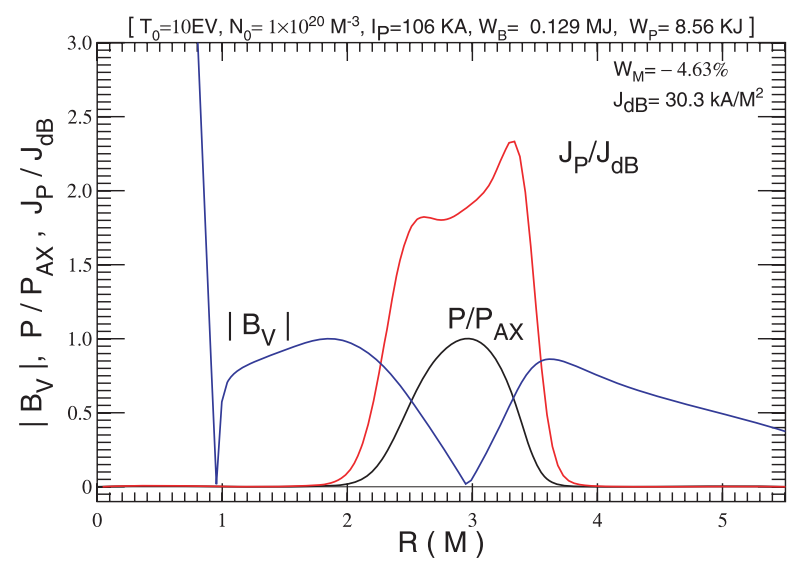

Fig. 5 Equilibrium profiles of a plasma pressure $P$ and toroidal current density $J_{\mathrm{p}}$ at $T_{0}=10 \mathrm{eV}$. The vertical magnetic field $B$ is reversed on the magnetic axis and inside of the center solenoid coil.

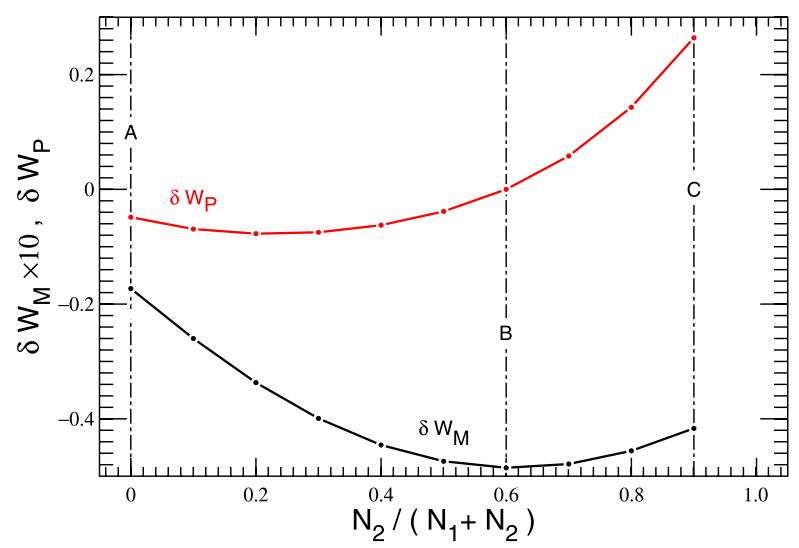

Fig. 6 Evolution of the equilibrium as a function of a density ratio $n_{1}$ and $n_{2}$. The MHD potential energy variation $\delta W_{\mathrm{M}}$ is minimized at $n_{2} /\left(n_{1}+n_{2}\right)=0.6$ indicated by chained line B. $\delta W_{\mathrm{p}}$ is the relative deviation of the stored plasma energy from the the minimum $W_{\mathrm{M}}$ state. In this case, $n_{3}=$ 0 .

tokamak plasma were calculated by minimizing the MHD potential energy; the results are shown in Figs. 5 and 6. The current profile is nearly centrally peaked. In this calculation, the four center solenoid coils carry the current $(-0.31$, $-0.26,-0.29$, and -0.32$) \mathrm{kA}$. The MHD potential energy is calculated while varying the density ratio of the centrally peaked current profile and the the current hole profile, as shown in Fig. 6. The relative deviation of the stored energy from the minimum $W_{\mathrm{M}}$ state profile is also plotted. $\delta W_{\mathrm{M}}$ becomes minimum at the appropriate density ratio indicated by chained line B. Therefore, to the left side of line $\mathrm{B}$, the variation of $\delta W_{\mathrm{M}}$ means that the tokamak plasma becomes more stable by shifting the current profile from the centrally peaked to hollow.

\section{Summary and Discussion}

A code utilizing a numerical integral equation based on the Biot-Savart law has been developed to analyze steady state tokamak equilibria by RF heating alone. In this calculation method, positions of the magnetic axis and two null points are specified. The six degrees of freedom of the three positions can be adjusted by controlling the currents in the six poloidal field coils; in order to apply this code, the target device must have at least six independently controllable poloidal field coils, like JT-60SA. Calculations have been performed using the poloidal field coil parameters for JT-60SA. Comparing $5 \mathrm{eV}$ (Fig. 1) and $10 \mathrm{keV}$ (Fig. 2) plasmas, the magnetic flux surfaces are almost identical when properly adjusting the coil currents.

In this calculation method, it is assumed that the plasma current is driven by the plasma pressure alone, without any other current drive mechanisms. A centrally peaked current distribution in a low-temperature stable plasma, as shown in Fig. 5, evolves into a current distribution with a hole, as shown in Fig. 4, by increasing the plasma energy. The stored energy $W_{\mathrm{P}}$ is increased by heating, that is one of the MHD instability factor. In order to stabilize the plasma, the magnetic potential energy $W_{\mathrm{B}}$ must be reduced by cancelling out the magnetic field of the external coil currents and plasma current (reduction of the magnetic energy $\left.W_{\mathrm{B}}\right), \delta W_{\mathrm{M}}=\delta\left(W_{\mathrm{P}}+W_{\mathrm{B}}\right)<0$. Figure 6 shows that the magnetic potential energy is decreased by enhancing the hollow current profile component. An example of the high temperature MHD stable plasma is shown in Figs. 3 and 4 with $\delta W_{\mathrm{M}}<0$ and current hole profile. This calculation method shows that the pressure driven current in tokamak plasmas forms a current distribution which reduces the MHD potential energy. The stable steady state tokamak should have a reconstructed current distribution which satisfies the condition of the minimum MHD potential energy. Under sufficient heating, the tokamak plasmas form a current hole which minimizes the MHD potential energy.

The Minimum MHD potential energy with the tokamak plasma shown in Fig. 6 is lower than that of the vacuum. The tokamak plasma with $\delta W_{\mathrm{M}}<0$ should be resistant to disruptions due to any perturbation. Therefore, in order to stabilize the tokamak equilibrium, the tokamak plasma must be heated until it reaches this stored plasma energy, which satisfies $\delta W_{\mathrm{M}}<0$. The steady state ratio $\left(n_{1}, n_{2}, n_{3}\right)$ of the plasma density distribution is determined by the condition of the minimum $\delta W_{\mathrm{M}}$.

The third, $n_{3}$ term in Eq. (12) is necessary for obtaining the current hole profile. M.S. Chu and P.B. Parks have studied the minimum of the MHD potential energy of the current hole and have found that it acts as if the current hole region were a vacuum region [5].

This calculation method can be utilized for experiment planning and a new tokamak design. The total current of EF coils for the $20 \mathrm{keV}$ plasma calculation shown in Fig. 3 is smaller than that for the $10 \mathrm{keV}$ plasma calculation shown in Fig. 2. In order to utilize the magnetic energy of EF coils efficiently, the center solenoid coils should be 
used. This may be relevant to an RF start-up experiment, by using a steady state center solenoid coil current.

\section{Acknowledgments}

The author is grateful to Professor Y. Takase and Professor A. Ejiri for giving me an opportunity to work with the TST2 experiments. Thanks to a great contribution for the paper modification by the good referee.

[1] C.B. Forest et al., Phys. Rev. Lett. 68, 3559 (1992).

[2] T. Maekawa et al., Nucl. Fusion 45, 1439 (2005).

[3] O. Watanabe et al., Plasma Fusion Res. 3, 026 (2008).

[4] T. Ichige et al., JAEA-Data/Code 2010-021.

[5] M.S. Chu et al., Phys. Plasmas 11, 4859 (2004). 